\title{
Certain fractional conformable inequalities for the weighted and the extended Chebyshev functionals
}

\author{
Asifa Tassaddiq ${ }^{1 *}$ (D), Gauhar Rahman², Kottakkaran Sooppy Nisar ${ }^{3}$ and Muhammad Samraiz ${ }^{4}$
}

\author{
"Correspondence: \\ a.tassaddiq@mu.edu.sa \\ 'Department of Basic Sciences and \\ Humanities, College of Computer \\ and Information Sciences, Majmaah \\ University, Al Majmaah, Saudi Arabia \\ Full list of author information is \\ available at the end of the article
}

\begin{abstract}
The main aim of this present paper is to establish fractional conformable inequalities for the weighted and extended Chebyshev functionals. We present some special cases of our main result in terms of the Riemann-Liouville fractional integral operator and classical inequalities.
\end{abstract}

MSC: Primary 26A33; secondary 26D10; 05A30

Keywords: Riemann-Liouville fractional integral; Fractional conformable integral; Chebyshev's functional; Extended Chebyshev functional; Weighted functional 
In [7], the Chebyshev functional for two integrable functions $f$ and $g$ on $[a, b]$ is defined as

$$
\mathcal{T}(f, g)=\frac{1}{b-a} \int_{a}^{b} f(\tau) g(\tau) d \tau-\frac{1}{b-a}\left(\int_{a}^{b} f(\tau) d \tau\right) \frac{1}{b-a}\left(\int_{a}^{b} g(\tau) d \tau\right) .
$$

In $[3,4,15,17]$, the applications and several inequalities related to (1) are found. In ([10], also see [7]), the Chebyshev functional is defined by

$$
\mathcal{T}(f, g, h)=\int_{a}^{b} h(\tau) d \tau \int_{a}^{b} h(\tau) f(\tau) g(\tau) d \tau-\int_{a}^{b} h(\tau) f(\tau) d \tau \int_{a}^{b} h(\tau) g(\tau) d \tau,
$$

where $f$ and $g$ are integrable on $[a, b]$ and $h$ is a positive and integrable function on $[a, b]$. Applications of the functional defined in (2) are found in probability and statistical problems. Further applications in differential and integral equations are found in $[6,16,31]$. Elezovic et al. [19] defined

$$
\begin{aligned}
|\mathcal{T}(f, g, h)| \leq & \frac{1}{2}\left(\left.\left.\int_{a}^{b} \int_{a}^{b} h(\theta) h(\vartheta)|\theta-\vartheta|^{\frac{1}{p^{\prime}}+\frac{1}{q^{\prime}}}\left|\int_{\vartheta}^{\theta}\right| f^{\prime}(\tau)\right|^{p} d \tau\right|^{\frac{r}{p}} d \theta d \vartheta\right)^{\frac{1}{r}} \\
& \times\left(\left.\left.\int_{a}^{b} \int_{a}^{b} h(\theta) h(\vartheta)|\theta-\vartheta|^{\frac{1}{p^{\prime}}+\frac{1}{q^{\prime}}}\left|\int_{\vartheta}^{\theta}\right| g^{\prime}(\tau)\right|^{q} d \tau\right|^{\frac{r}{q}} d \theta d \vartheta\right)^{\frac{1}{r^{\prime}}} \\
\leq & \frac{1}{2}\left\|f^{\prime}\right\|\left\|g^{\prime}\right\|\left(\int_{a}^{b} \int_{a}^{b} h(\theta) h(\vartheta)|\theta-\vartheta|^{\frac{1}{p^{\prime}}+\frac{1}{q^{\prime}}} d \theta d \vartheta\right)
\end{aligned}
$$

where $f^{\prime} \in L^{p}([a, b]), g^{\prime} \in L^{q}([a, b]), p, q, r>1, \frac{1}{p}+\frac{1}{p^{\prime}}=1, \frac{1}{q}+\frac{1}{q^{\prime}}=1$ and $\frac{1}{r}+\frac{1}{r^{\prime}}=1$. In [13], the authors defined the following fractional integral inequality for Chebyshev functionals:

$$
\begin{aligned}
& 2\left|\mathcal{I}^{\alpha} h(\tau) \mathcal{I}^{\alpha} h f g(\tau)-\mathcal{I}^{\alpha} h f(\tau) \mathcal{I}^{\alpha} h g(\tau)\right| \\
& \quad \leq \frac{\left\|f^{\prime}\right\|_{p}\left\|g^{\prime}\right\|_{q}}{\Gamma^{2}(\alpha)} \int_{0}^{\tau} \int_{0}^{\tau}(\tau-\theta)^{\alpha-1}(\tau-\vartheta)^{\alpha-1}|\theta-\vartheta| h(\theta) h(\vartheta) d \theta d \vartheta,
\end{aligned}
$$

where $f^{\prime} \in L^{p}\left(\left[0, \infty[), g^{\prime} \in L^{q}\left(\left[0, \infty[), p, q>1, \frac{1}{p}+\frac{1}{q}=1\right.\right.\right.\right.$.

Let us consider the extended Chebyshev functional $[8,30]$

$$
\begin{aligned}
\tilde{\mathcal{T}}\left(f, g, h, h^{\prime}\right)= & \int_{a}^{b} h^{\prime}(\tau) d \tau \int_{a}^{b} h(\tau) f(\tau) g(\tau) d \tau+\int_{a}^{b} h(\tau) d \tau \int_{a}^{b} h^{\prime}(\tau) f(\tau) g(\tau) d \tau \\
& -\int_{a}^{b} h(\tau) f(\tau) d \tau \int_{a}^{b} h^{\prime}(\tau) g(\tau) d \tau \\
& -\int_{a}^{b} h^{\prime}(\tau) f(\tau) d \tau \int_{a}^{b} h(\tau) g(\tau) d \tau .
\end{aligned}
$$

In $[4,11,29]$, various researchers have addressed the functionals (2) and (5). Recently Rahman et al. [38] defined fractional conformable inequalities for Chebyshev functionals (1) and (2). The present paper aims to develop certain fractional conformable inequalities for the Chebyshev functionals (2) and (5). Also, we will discuss some particular cases of our main result. 


\section{Preliminaries}

In this section, we present the following well-known definitions from [20, 23].

Definition 2.1 The Riemann-Liouville fractional integral $\mathfrak{I}_{a^{+}}^{\alpha}$ and $\mathfrak{I}_{b^{-}}^{\alpha}$ of order $\alpha>0$, for a continuous function $f \in[a, b]$, is defined by

$$
\Im_{a}^{\alpha} f(x)=\frac{1}{\Gamma(\alpha)} \int_{0}^{\tau}(\tau-t)^{\alpha-1} f(t) d t, \quad a<\tau \leq b,
$$

where $\Gamma$ is the gamma function; for further details as regards gamma and related functions, see [45].

Definition 2.2 The fractional conformable integral ${ }^{\beta} \mathfrak{I}_{0}^{\alpha}$ of order $\beta>0$, for a continuous function is defined by

$$
{ }^{\beta} \mathfrak{I}_{0}^{\alpha} f(\tau)=\frac{1}{\Gamma(\beta)} \int_{a}^{\tau}\left(\frac{\tau^{\alpha}-t^{\alpha}}{\alpha}\right)^{\beta-1} \frac{f(t)}{t^{1-\alpha}} d t ; \quad 0<\tau \leq b .
$$

Clearly one can get ${ }^{0} \mathfrak{I}_{0}^{\alpha} f(\tau)=f(\tau)$ and

$$
{ }^{\beta} \mathfrak{I}_{0}^{\alpha \gamma} \mathfrak{I}_{0}^{\alpha} f(\tau)={ }^{\beta+\gamma} \mathfrak{I}_{0}^{\alpha} f(\tau)={ }^{\gamma} \mathfrak{I}_{0}^{\alpha \beta} \mathfrak{I}_{0}^{\alpha} f(\tau) ; \quad \beta, \lambda>0 .
$$

In $[23,35,37,38,40,43]$, one has studied fractional conformable integral operators and has established certain inequalities by employing the said fractional integral operators.

Remark 1 If we consider $\alpha=1$, then (7) will lead to the fractional integral in (6).

\section{Main results}

In this section, we establish certain fractional conformable inequalities for the weighted and the extended Chebyshev functionals.

Theorem 3.1 Let $f$ and $g$ be two differentiable functions on $[0, \infty)$ and let $h$ be positive and integrable function on $[0, \infty)$. If $f^{\prime} \in L^{p}\left(\left[0, \infty[), g^{\prime} \in L^{q}\left(\left[0, \infty[), p, q, r>1\right.\right.\right.\right.$ with $\frac{1}{p}+\frac{1}{p^{\prime}}=1$, $\frac{1}{q}+\frac{1}{q^{\prime}}=1$ and $\frac{1}{r}+\frac{1}{r^{\prime}}=1$, then the following inequality holds for all $\tau>0, \alpha, \beta>0$ :

$$
\begin{aligned}
\left.2\right|^{\beta} \mathfrak{I}^{\alpha} h(\tau)^{\beta} \mathfrak{I}^{\alpha} h f g(\tau)-{ }^{\beta} \mathfrak{I}^{\alpha} h f(\tau)^{\beta} \mathfrak{I}^{\alpha} h g(\tau) \mid & \\
\leq & \left(\frac{\left\|f^{\prime}\right\|_{p}^{r}}{\Gamma(\beta)} \int_{0}^{\tau} \int_{0}^{\tau}\left(\frac{\tau^{\alpha}-\theta^{\alpha}}{\alpha}\right)^{\beta-1}\left(\frac{\tau^{\alpha}-\vartheta^{\alpha}}{\alpha}\right)^{\beta-1}\right. \\
& \left.\times \theta^{\alpha-1} \vartheta^{\alpha-1} h(\theta) h(\vartheta)|\theta-\vartheta|^{\frac{1}{p^{\prime}}+\frac{1}{q^{\prime}}} d \theta d \vartheta\right)^{\frac{1}{r}} \\
& \times\left(\frac{\left\|g^{\prime}\right\|_{q}^{r^{\prime}}}{\Gamma(\beta)} \int_{0}^{\tau} \int_{0}^{\tau}\left(\frac{\tau^{\alpha}-\theta^{\alpha}}{\alpha}\right)^{\beta-1}\left(\frac{\tau^{\alpha}-\vartheta^{\alpha}}{\alpha}\right)^{\beta-1}\right. \\
& \left.\times \theta^{\alpha-1} \vartheta^{\alpha-1} h(\theta) h(\vartheta)|\theta-\vartheta|^{\frac{1}{p^{\prime}}}+\frac{1}{q^{\prime}} d \theta d \vartheta\right)^{\frac{1}{r^{\prime}}}
\end{aligned}
$$




$$
\begin{aligned}
\leq & \frac{\left\|f^{\prime}\right\|_{\alpha}^{r}\left\|g^{\prime}\right\|_{q}^{r^{\prime}}}{\Gamma^{2}(\beta)}\left(\int_{0}^{\tau} \int_{0}^{\tau}\left(\frac{\tau^{\alpha}-\theta^{\alpha}}{\alpha}\right)^{\beta-1}\left(\frac{\tau^{\alpha}-\vartheta^{\alpha}}{\alpha}\right)^{\beta-1}\right. \\
& \left.\times \theta^{\alpha-1} \vartheta^{\alpha-1} h(\theta) h(\vartheta)|\theta-\vartheta|^{\frac{1}{p^{\prime}}+\frac{1}{q^{\prime}}} d \theta d \vartheta\right) .
\end{aligned}
$$

Proof Let us define

$$
H(\theta, \vartheta)=(f(\theta)-f(\vartheta))(g(\theta)-g(\vartheta)) ; \quad \theta, \vartheta \in(0, \tau)
$$

Multiplying (9) by $\frac{1}{\Gamma(\beta)}\left(\frac{\tau^{\alpha}-\theta^{\alpha}}{\alpha}\right)^{\beta-1} \theta^{\alpha-1} h(\theta)$ and then integrating with respect to $\theta$ over $(0, \tau)$, we have

$$
\begin{aligned}
& \frac{1}{\Gamma(\beta)} \int_{0}^{\tau}\left(\frac{\tau^{\alpha}-\theta^{\alpha}}{\alpha}\right)^{\beta-1} \theta^{\alpha-1} h(\theta) H(\theta, \vartheta) d \theta \\
& \quad={ }^{\beta} \mathfrak{I}^{\alpha} h f g(\tau)-g(\vartheta)^{\beta} \mathfrak{I}^{\alpha} h f(\tau)-f(\theta)^{\beta} \mathfrak{I}^{\alpha} h g(\tau)+f(\vartheta) g(\vartheta)^{\beta} \mathfrak{I}^{\alpha} h(\tau) .
\end{aligned}
$$

Again, multiplying (10) by $\frac{1}{\Gamma(\beta)}\left(\frac{\tau^{\alpha}-\vartheta^{\alpha}}{\alpha}\right)^{\beta-1} \vartheta^{\alpha-1} h(\vartheta)$ and then integrating with respect to $v$ over $(0, \tau)$, we have

$$
\begin{aligned}
& \frac{1}{\Gamma^{2}(\beta)} \int_{0}^{\tau} \int_{0}^{\tau}\left(\frac{\tau^{\alpha}-\theta^{\alpha}}{\alpha}\right)^{\beta-1} \theta^{\alpha-1}\left(\frac{\tau^{\alpha}-\vartheta^{\alpha}}{\alpha}\right)^{\beta-1} \vartheta^{\alpha-1} h(\theta) h(\vartheta) H(\theta, \vartheta) d \theta d \vartheta \\
& =2\left({ }^{\beta} \mathfrak{I}^{\alpha} h(\tau)^{\beta} \mathfrak{I}^{\alpha} h f g(\tau)-{ }^{\beta} \mathfrak{I}^{\alpha} h f(\tau)^{\beta} \mathfrak{I}^{\alpha} h g(\tau)\right) .
\end{aligned}
$$

Also, on the other hand, we have

$$
H(\theta, \vartheta)=\int_{\vartheta}^{\theta} \int_{\vartheta}^{\theta} f^{\prime}(x) g^{\prime}(y) d x d y
$$

By employing the Hölder inequality, we have

$$
|f(\theta)-f(\vartheta)| \leq\left.\left.|\theta-\vartheta|^{\frac{1}{p^{\prime}}}\left|\int_{\vartheta}^{\theta}\right| f^{\prime}(x)\right|^{p} d x\right|^{\frac{1}{p}}
$$

and

$$
|g(\theta)-g(\vartheta)| \leq\left.\left.|\theta-\vartheta|^{\frac{1}{q^{\prime}}}\left|\int_{\vartheta}^{\theta}\right| g^{\prime}(y)\right|^{q} d y\right|^{\frac{1}{q}}
$$

Then $H$ becomes

$$
|H(\theta, \vartheta)| \leq\left.\left.\left.\left.|\theta-\vartheta|^{\frac{1}{p^{\prime}}+\frac{1}{q^{\prime}}}\left|\int_{\theta}^{\vartheta}\right| f^{\prime}(x)\right|^{p} d x\right|^{\frac{1}{p}}\left|\int_{\vartheta}^{\theta}\right| g^{\prime}(\tau)\right|^{q} d y\right|^{\frac{1}{q}} .
$$

Therefore, from (11) and (15), we can write

$$
\begin{aligned}
\left.2\right|^{\beta} \mathfrak{I}^{\alpha} h(\tau)^{\beta} \mathfrak{I}^{\alpha} h f g(\tau)-{ }^{\beta} \mathfrak{I}^{\alpha} h f(\tau)^{\beta} \mathfrak{I}^{\alpha} h g(\tau) \mid \\
\quad=\frac{1}{\Gamma^{2}(\beta)} \int_{0}^{\tau} \int_{0}^{\tau}\left(\frac{\tau^{\alpha}-\theta^{\alpha}}{\alpha}\right)^{\beta-1} \theta^{\alpha-1}\left(\frac{\tau^{\alpha}-\vartheta^{\alpha}}{\alpha}\right)^{\beta-1} \vartheta^{\alpha-1} h(\theta) h(\vartheta)|H(\theta, \vartheta)| d \theta d \vartheta
\end{aligned}
$$




$$
\begin{aligned}
\leq & \frac{1}{\Gamma^{2}(\beta)} \int_{0}^{\tau} \int_{0}^{\tau}\left(\frac{\tau^{\alpha}-\theta^{\alpha}}{\alpha}\right)^{\beta-1} \theta^{\alpha-1}\left(\frac{\tau^{\alpha}-\vartheta^{\alpha}}{\alpha}\right)^{\beta-1} \vartheta^{\alpha-1} h(\theta) h(\vartheta) \\
& \times\left.\left.\left.\left.|\theta-\vartheta|^{\frac{1}{p^{\prime}}+\frac{1}{q^{\prime}}}\left|\int_{\vartheta}^{\theta}\right| f^{\prime}(x)\right|^{p} d x\right|^{\frac{1}{p}}\left|\int_{\vartheta}^{\theta}\right| g^{\prime}(\tau)\right|^{q} d t\right|^{\frac{1}{q}} d \theta d \vartheta .
\end{aligned}
$$

Now, by using the Hölder inequality for the double integral, we have

$$
\begin{aligned}
&\left.2\right|^{\beta} \mathfrak{I}^{\alpha} h(\tau)^{\beta} \mathfrak{I}^{\alpha} h f g(\tau)-{ }^{\beta} \mathfrak{I}^{\alpha} h f(\tau)^{\beta} \mathfrak{I}^{\alpha} h g(\tau) \mid \\
& \leq \frac{1}{\Gamma^{2}(\beta)}\left(\int_{0}^{\tau} \int_{0}^{\tau}\left(\frac{\tau^{\alpha}-\theta^{\alpha}}{\alpha}\right)^{\beta-1} \theta^{\alpha-1}\left(\frac{\tau^{\alpha}-\vartheta^{\alpha}}{\alpha}\right){ }^{\beta-1}\right. \\
&\left.\times\left.\left.\vartheta^{\alpha-1} h(\theta) h(\vartheta)|\theta-\vartheta|^{\frac{1}{p^{\prime}}+\frac{1}{q^{\prime}}}\left|\int_{\vartheta}^{\theta}\right| f^{\prime}(x)\right|^{p} d x\right|^{\frac{r}{p}} d \theta d \vartheta\right)^{\frac{1}{r}} \\
& \times\left(\int_{0}^{\tau} \int_{0}^{\tau}\left(\frac{\tau^{\alpha}-\theta^{\alpha}}{\alpha}\right)^{\beta-1} \theta^{\alpha-1}\left(\frac{\tau^{\alpha}-\vartheta^{\alpha}}{\alpha}\right)^{\beta-1}\right. \\
&\left.\times\left.\left.\vartheta^{\alpha-1} h(\theta) h(\vartheta)|\theta-\vartheta|^{\frac{1}{p^{\prime}}+\frac{1}{q^{\prime}}}\left|\int_{\vartheta}^{\theta}\right| g^{\prime}(x)\right|^{p} d x\right|^{\frac{r^{\prime}}{q}} d \theta d \vartheta\right)^{\frac{1}{r^{\prime}}} .
\end{aligned}
$$

Now, using the following properties:

$$
\left.\left|\int_{\vartheta}^{\theta}\right| f^{\prime}(x)\right|^{p} d x\left|\leq\left\|f^{\prime}\right\|_{p}^{p},\right| \int_{\vartheta}^{\theta}\left|g^{\prime}(y)\right|^{q} d y \mid \leq\left\|g^{\prime}\right\|_{q^{\prime}}^{q},
$$

(17) can be written as

$$
\begin{aligned}
&\left.2\right|^{\beta} \mathfrak{I}^{\alpha} h(\tau)^{\beta} \mathfrak{I}^{\alpha} h f g(\tau)-{ }^{\beta} \mathfrak{I}^{\alpha} h f(\tau)^{\beta} \mathfrak{I}^{\alpha} h g(\tau) \mid \\
& \leq\left(\frac{\left\|f^{\prime}\right\|_{p}^{r}}{\Gamma^{r}(\beta)} \int_{0}^{\tau} \int_{0}^{\tau}\left(\frac{\tau^{\alpha}-\theta^{\alpha}}{\alpha}\right)^{\beta-1} \theta^{\alpha-1}\left(\frac{\tau^{\alpha}-\vartheta^{\alpha}}{\alpha}\right)^{\beta-1}\right. \\
&\left.\times \vartheta^{\alpha-1} h(\theta) h(\vartheta)|\theta-\vartheta|^{\frac{1}{p^{\prime}}+\frac{1}{q^{\prime}}} \mid d \theta d \vartheta\right)^{\frac{1}{r}} \\
& \times\left(\frac{\left\|g^{\prime}\right\|_{q}^{r^{\prime}}}{\Gamma^{r^{\prime}}(\beta)} \int_{0}^{\tau} \int_{0}^{\tau}\left(\frac{\tau^{\alpha}-\theta^{\alpha}}{\alpha}\right)^{\beta-1} \theta^{\alpha-1}\left(\frac{\tau^{\alpha}-\vartheta^{\alpha}}{\alpha}\right)^{\beta-1}\right. \\
&\left.\times \vartheta^{\alpha-1} h(\theta) h(\vartheta)|\theta-\vartheta|^{\frac{1}{p^{\prime}}+\frac{1}{q^{\prime}}} d \theta d \vartheta\right)^{\frac{1}{r^{\prime}}} .
\end{aligned}
$$

Therefore,

$$
\begin{aligned}
\left.2\right|^{\beta} \mathfrak{I}^{\alpha} h(\tau)^{\beta} \mathfrak{I}^{\alpha} h f g(\tau)-{ }^{\beta} \mathfrak{I}^{\alpha} h f(\tau)^{\beta} \mathfrak{I}^{\alpha} h g(\tau) \mid \\
\leq \frac{\left\|f^{\prime}\right\|_{\alpha}^{r}\left\|g^{\prime}\right\|_{q}^{r^{\prime}}}{\Gamma^{2}(\beta)}\left(\int_{0}^{\tau} \int_{0}^{\tau}\left(\frac{\tau^{\alpha}-\theta^{\alpha}}{\alpha}\right)^{\beta-1}\left(\frac{\tau^{\alpha}-\vartheta^{\alpha}}{\alpha}\right)^{\beta-1}\right. \\
\left.\quad \times \theta^{\alpha-1} \vartheta^{\alpha-1} h(\theta) h(\vartheta)|\theta-\vartheta|^{\frac{1}{p^{\prime}}+\frac{1}{q^{\prime}}} d \theta d \vartheta\right)
\end{aligned}
$$

which gives the required proof. 
By considering $\alpha=1$ in Theorem 3.1, we get the following well-known result of Dahmani et al. [12].

Corollary 1 Let $f$ and $g$ be two differentiable functions on $[0, \infty)$ and let $h$ be positive and integrable function on $[0, \infty)$. If $f^{\prime} \in L^{p}\left(\left[0, \infty[), g^{\prime} \in L^{q}\left(\left[0, \infty[), p, q, r>1\right.\right.\right.\right.$ with $\frac{1}{p}+\frac{1}{p^{\prime}}=1$, $\frac{1}{q}+\frac{1}{q^{\prime}}=1$ and $\frac{1}{r}+\frac{1}{r^{\prime}}=1$, then the following inequality holds for all $\tau>0, \beta>0$ :

$$
\begin{aligned}
2\left|\mathfrak{I}^{\beta} h(\tau) \mathfrak{I}^{\beta} h f g(\tau)-\mathfrak{I}^{\beta} h f(\tau) \mathfrak{I}^{\beta} h g(\tau)\right| & \\
\leq & \left(\frac{\left\|f^{\prime}\right\|_{p}^{r}}{\Gamma(\beta)} \int_{0}^{\tau} \int_{0}^{\tau}(\tau-\theta)^{\beta-1}(\tau-\vartheta)^{\beta-1} h(\theta) h(\vartheta)|\theta-\vartheta|^{\frac{1}{p^{\prime}}+\frac{1}{q^{\prime}}} d \theta d \vartheta\right)^{\frac{1}{r}} \\
& \times\left(\frac{\left\|g^{\prime}\right\|_{q}^{r^{\prime}}}{\Gamma(\beta)} \int_{0}^{\tau} \int_{0}^{\tau}(\tau-\theta)^{\beta-1}(\tau-\vartheta)^{\beta-1} h(\theta) h(\vartheta)|\theta-\vartheta|^{\frac{1}{p^{\prime}}+\frac{1}{q^{\prime}}} d \theta d \vartheta\right)^{\frac{1}{r^{\prime}}} \\
\leq & \frac{\left\|f^{\prime}\right\|_{\alpha}^{r}\left\|g^{\prime}\right\|_{q}^{r^{\prime}}}{\Gamma^{2}(\beta)}\left(\int_{0}^{\tau} \int_{0}^{\tau}(\tau-\theta)^{\beta-1}(\tau-\vartheta)^{\beta-1} h(\theta) h(\vartheta)|\theta-\vartheta|^{\frac{1}{p^{\prime}}+\frac{1}{q^{\prime}}} d \theta d \vartheta\right) .
\end{aligned}
$$

Remark 2 Similarly, by considering $\alpha=\beta=1$ in Theorem 3.1, we get the inequality (3).

Theorem 3.2 Let $f$ and $g$ be two differentiable functions on $[0, \infty)$ and let $h$ and $h^{\prime}$ be positive and integrable functions on $[0, \infty)$. If $f^{\prime} \in L^{p}\left(\left[0, \infty[), g^{\prime} \in L^{q}([0, \infty[), p, q, r>1\right.\right.$ with $\frac{1}{p}+\frac{1}{p^{\prime}}=1, \frac{1}{q}+\frac{1}{q^{\prime}}=1$ and $\frac{1}{r}+\frac{1}{r^{\prime}}=1$, then the following inequality holds for all $\tau>0$, $\alpha, \beta>0$ :

$$
\begin{aligned}
& \mid{ }^{\beta} \mathfrak{I}^{\alpha} h^{\prime}(\tau)^{\beta} \mathfrak{I}^{\alpha} h f g(\tau)+{ }^{\beta} \mathfrak{I}^{\alpha} h(\tau)^{\beta} \mathfrak{I}^{\alpha} h^{\prime} f g(\tau)-{ }^{\beta} \mathfrak{I}^{\alpha} h f(\tau)^{\beta} \mathfrak{I}^{\alpha} h^{\prime} g(\tau) \\
& \quad-{ }^{\beta} \mathfrak{I}^{\alpha} h^{\prime} f(\tau)^{\beta} \mathfrak{I}^{\alpha} h g(\tau) \mid \\
& \leq \frac{\left\|f^{\prime}\right\|_{\alpha}^{r}\left\|g^{\prime}\right\|_{q}^{r^{\prime}}}{\Gamma^{2}(\beta)} \int_{0}^{\tau} \int_{0}^{\tau}\left(\frac{\tau^{\alpha}-\theta^{\alpha}}{\alpha}\right)^{\beta-1}\left(\frac{\tau^{\alpha}-\vartheta^{\alpha}}{\alpha}\right)^{\beta-1} \\
& \quad \times \theta^{\alpha-1} \vartheta^{\alpha-1} h(\theta) h^{\prime}(\vartheta)|\theta-\vartheta|^{\frac{1}{p^{\prime}}+\frac{1}{q^{\prime}}} d \theta d \vartheta .
\end{aligned}
$$

Proof Multiplying (10) by $\frac{1}{\Gamma(\beta)}\left(\frac{\tau^{\alpha}-\vartheta^{\alpha}}{\alpha}\right)^{\mu-1} \vartheta^{\alpha-1} h(\vartheta)$ and then integrating with respect to $\vartheta$ over $(0, \tau)$, we have

$$
\begin{gathered}
\frac{1}{\Gamma^{2}(\beta)} \int_{0}^{\tau} \int_{0}^{\tau}\left(\frac{\tau^{\alpha}-\theta^{\alpha}}{\alpha}\right)^{\beta-1} \theta^{\alpha-1}\left(\frac{\tau^{\alpha}-\vartheta^{\alpha}}{\alpha}\right)^{\mu-1} \vartheta^{\alpha-1} h(\theta) h(\vartheta) H(\theta, \vartheta) d \theta d \vartheta \\
={ }^{\beta} \mathfrak{I}^{\alpha} h^{\prime}(\tau)^{\beta} \mathfrak{I}^{\alpha} h f g(\tau)+{ }^{\beta} \mathfrak{I}^{\alpha} h(\tau)^{\beta} \mathfrak{I}^{\alpha} h^{\prime} f g(\tau) \\
-{ }^{\beta} \mathfrak{I}^{\alpha} h f(\tau)^{\beta} \mathfrak{I}^{\alpha} h^{\prime} g(\tau)-{ }^{\beta} \mathfrak{I}^{\alpha} h^{\prime} f(\tau)^{\beta} \mathfrak{I}^{\alpha} h g(\tau) .
\end{gathered}
$$

Using (15) in (22), we obtain

$$
\begin{aligned}
\mid{ }^{\beta} \mathfrak{I}^{\alpha} h^{\prime}(\tau)^{\beta} \mathfrak{I}^{\alpha} h f g(\tau)+{ }^{\beta} \mathfrak{I}^{\alpha} h(\tau)^{\beta} \mathfrak{I}^{\alpha} h^{\prime} f g(\tau)-{ }^{\beta} \mathfrak{I}^{\alpha} h f(\tau)^{\beta} \mathfrak{I}^{\alpha} h^{\prime} g(\tau) \\
\quad-{ }^{\beta} \mathfrak{I}^{\alpha} h^{\prime} f(\tau){ }^{\beta} \mathfrak{I}^{\alpha} h g(\tau) \mid \\
=\frac{1}{\Gamma(\beta) \Gamma(\mu)} \int_{0}^{\tau} \int_{0}^{\tau}\left(\frac{\tau^{\alpha}-\theta^{\alpha}}{\alpha}\right)^{\beta-1} \vartheta^{\alpha-1}\left(\frac{\tau^{\alpha}-\vartheta^{\alpha}}{\alpha}\right)^{\beta-1} \vartheta^{\alpha-1} h(\theta) h^{\prime}|H(\theta, \vartheta)| d \theta d \vartheta
\end{aligned}
$$




$$
\begin{aligned}
\leq & \frac{\left\|f^{\prime}\right\|^{\alpha}\left\|g^{\prime}\right\|^{\alpha}}{\Gamma^{2}(\beta)} \int_{0}^{\tau} \int_{0}^{\tau}\left(\frac{\tau^{\alpha}-\theta^{\alpha}}{\alpha}\right)^{\beta-1} \theta^{\alpha-1}\left(\frac{\tau^{\alpha}-\vartheta^{\alpha}}{\alpha}\right){ }^{\mu-1} \vartheta^{\alpha-1}|\theta-\vartheta|^{\frac{1}{p^{\prime}}+\frac{1}{q^{\prime}}} \\
& \times\left.\left.\left.\left.\left|\int_{\theta}^{\vartheta}\right| f^{\prime}(x)\right|^{p} d x\right|^{\frac{1}{p}}\left|\int_{\vartheta}^{\theta}\right| g^{\prime}(\tau)\right|^{q} d t\right|^{\frac{1}{q}} h(\theta) h^{\prime}(\vartheta) d \theta d \vartheta .
\end{aligned}
$$

Applying the similar procedure of Theorem 3.1, we obtain the desired proof.

If we consider $\alpha=1$ in Theorem 3.2, then we get the following well-known result [12].

Corollary 2 Let $f$ and $g$ be two differentiable functions on $[0, \infty)$ and let $h$ and $h^{\prime}$ be positive and integrable functions on $[0, \infty)$. Iff $\in L^{p}\left(\left[0, \infty[), g^{\prime} \in L^{q}\left(\left[0, \infty[), p, q, r>1\right.\right.\right.\right.$ with $\frac{1}{p}+\frac{1}{p^{\prime}}=$ $1, \frac{1}{q}+\frac{1}{q^{\prime}}=1$ and $\frac{1}{r}+\frac{1}{r^{\prime}}=1$, then the following inequality holds for all $\tau>0, \beta>0$ :

$$
\begin{aligned}
\left|\mathfrak{I}^{\beta} h^{\prime}(\tau) \mathfrak{I}^{\beta} h f g(\tau)+\mathfrak{I}^{\beta} h(\tau) \mathfrak{I}^{\beta} h^{\prime} f g(\tau)-\mathfrak{I}^{\beta} h f(\tau) \mathfrak{I}^{\beta} h^{\prime} g(\tau)-\mathfrak{I}^{\beta} h^{\prime} f(\tau) \mathfrak{I}^{\beta} h g(\tau)\right| \\
\leq \frac{\left\|f^{\prime}\right\|_{\alpha}^{r}\left\|g^{\prime}\right\|_{q}^{r^{\prime}}}{\Gamma^{2}(\beta)} \int_{0}^{\tau} \int_{0}^{\tau}(\tau-\theta)^{\beta-1}(\tau-\vartheta)^{\beta-1} \\
\quad \times \theta^{\alpha-1} \vartheta^{\alpha-1} h(\theta) h^{\prime}(\vartheta)|\theta-\vartheta|^{\frac{1}{p^{\prime}}+\frac{1}{q^{\prime}}} d \theta d \vartheta .
\end{aligned}
$$

Remark 3 If we let $\beta=\alpha=1$ in Theorem 3.2, then we get the inequality (4).

\section{Concluding remarks}

In this paper, we established certain fractional conformable inequalities related to the weighted and the extended Chebyshev functionals. The inequalities obtained in the present paper are more general than the existing classical inequalities cited therein. This work will reduce to the inequalities some Riemann-Liouville integral inequalities by taking $\alpha=1$, which have been presented earlier by [12]. Also, one can get the classical results by taking $\alpha=\beta=1$.

\section{Acknowledgements}

Asifa Tassaddiq would like to thank Deanship of Scientific Research at Majmaah University, for supporting this work under Project Number (R-1441-74). The authors are thankful to the anonymous reviewers and editors for their useful suggestions and comments, significantly improving the quality of this manuscript.

Funding

None.

Availability of data and materials

Not applicable.

Competing interests

The authors declare that they have no competing interests.

Authors' contributions

All authors contributed equally. All authors read and approved the final manuscript.

Author details

1 Department of Basic Sciences and Humanities, College of Computer and Information Sciences, Majmaah University, Al Majmaah, Saudi Arabia. ${ }^{2}$ Department of Mathematics, Shaheed Benazir Bhutto University, Sheringal, Pakistan.

${ }^{3}$ Department of Mathematics, College of Arts and Sciences, Prince Sattam Bin Abdulaziz University, Wadi Aldawasir, Saudi Arabia. ${ }^{4}$ Department of Mathematics, University of Sargodha, Sargodha, Pakistan.

\section{Publisher's Note}

Springer Nature remains neutral with regard to jurisdictional claims in published maps and institutional affiliations. 


\section{References}

1. Abdeljawad, T.: On conformable fractional calculus. J. Comput. Appl. Math. 279, 57-66 (2015). https://doi.org/10.1016/j.cam.2014.10.016

2. Akdemir, A.O., Ekinci, A., Set, E.: Conformable fractional integrals and related new integral inequalities. J. Nonlinear Convex Anal. 18(4), 661-674 (2017)

3. Anastassiou, G., Hooshmandasl, M.R., Ghasemi, A., Moftakharzadeh, F.: Montgomery identities for fractional integrals and related fractional inequalities. J. Inequal. Pure Appl. Math. 10(4), Article 97 (2009)

4. Belarbi, S., Dahmani, Z.: On some new fractional integral inequalities. J. Inequal. Pure Appl. Math. 10(3), Article 86 (2009)

5. Budak, H., Pehlivan, E., Sarikaya, M.Z.: On generalized weighted fractional inequalities. Turk. J. Inequal. 3(2), 34-52 (2019)

6. Cerone, P., Dragomir, S.S.: A refinement of the Gruss inequality and applications. Tamkang J. Math. 38(1), 37-49 (2007)

7. Chebyshev, P.L.: Sur les expressions approximatives des integrales definies par les autres prises entre les mêmes limites. Proc. Math. Soc. Charkov 2, 93-98 (1882)

8. Dahmani, Z.: New inequalities in fractional integrals. Int. J. Nonlinear Sci. 9(4), 493-497 (2010)

9. Dahmani, Z:: New inequalities for a class of differentiable functions. Int. J. Nonlinear Anal. Appl. 2(2), 19-23 (2011)

10. Dahmani, Z.: The Riemann-Liouville operator to generate some new inequalities. Int. J. Nonlinear Sci. 12,452-455 (2011)

11. Dahmani, Z:: About some integral inequalities using Riemann-Liouville integrals. Gen. Math. 20(4), $63-69$ (2012)

12. Dahmani, Z., Khameli, A., Fareha, K.: Some RL-integral inequalities for the weighted and the extended Chebyshev functionals. Konuralp J. Math. 5(1), 43-48 (2017)

13. Dahmani, Z., Mechouar, O., Brahami, S.: Certain inequalities related to the Chebyshev's functional involving Riemann-Liouville operator. Bull. Math. Anal. Appl. 3(4), 38-44 (2011)

14. Dahmani, Z., Tabharit, L.: On weighted Gruss type inequalities via fractional integration. J. Adv. Res. Pure Math. 2(4), 31-38 (2010)

15. Dahmani, Z., Tabharit, I., Taf, S.: New inequalities via Riemann-Liouville fractional integration. J. Adv. Res. Sci. Comput. 2(1), 40-45 (2010)

16. Dragomir, S.S.: A generalization of Gruss's inequality in inner product spaces and applications. J. Math. Anal. Appl. 237(1), 74-82 (1999)

17. Dragomir, S.S.: Some integral inequalities of Gruss type. Indian J. Pure Appl. Math. 31(4), 397-415 (2002)

18. Ekinci, A., Özdemir, M.E.: Some new integral inequalities via Riemann-Liouville integral operators. Appl. Comput. Math. 18(3), 288-295 (2019)

19. Elezovic, N., Marangunic, L., Pecaric, G.: Some improvement of Grüss type inequality. J. Math. Inequal. 1(3), 425-436 (2007)

20. Gorenflo, R., Mainardi, F.: Fractional calculus, integral and differential equations of fractional order. In: Fractals and Fractional Calculus in Continuum Mechanics, pp. 223-276. Springer, Wien (1997)

21. Herrmann, R.: Fractional Calculus: An Introduction for Physicists. World Scientific, Singapore (2011)

22. Huang, C.J., Rahman, G., Nisar, K.S., Ghaffar, A., Qi, F.: Some inequalities of Hermite-Hadamard type for $k$-fractional conformable integrals. Aust. J. Math. Anal. Appl. 16(1), 1-9 (2019)

23. Jarad, F., Uurlu, E., Abdeljawad, T., Baleanu, D.: On a new class of fractional operators. Adv. Differ. Equ. 2017(1), 247 (2017). https://doi.org/10.1186/s13662-017-1306-z

24. Kashuri, A., Liko, R.: Ostrowski type conformable fractional integrals for generalized $(g, s, m, \phi)$-preinvex functions. Turk. J. Inequal. 2(2), 54-70 (2018)

25. Katugampola, U.N.: A new approach to generalized fractional derivatives. Bull. Math. Anal. Appl. 6, 1-15 (2014)

26. Katugampola, U.N.: New fractional integral unifying six existing fractional integrals (2016). arXiv:1612.08596

27. Khalil, R., Al Horani, M., Yousef, A., Sababheh, M.: A new definition of fractional derivative. J. Comput. Appl. Math. 264(65), 65-70 (2014)

28. Kilbas, A.A., Srivastava, H.M., Trujillo, J.J.: Theory and Applications of Fractional Differential Equations. North-Holland Mathematics Studies, vol. 207. Elsevier, Amsterdam (2006)

29. Mercer, A.McD.: An improvement of the Gruss inequality J. Inequal. Pure Appl. Math. 10(4), Article 93 (2005)

30. Mitrinovic, D.S.: Analytic Inequalities. Springer, Berlin (1970)

31. Mitrinovic, D.S., Pecaric, J.E., Fink, A.M.: Classical and New Inequalities in Analysis. Kluwer Academic, Dordrecht (1993)

32. Nisar, K.S., Rahman, G., Khan, A., Tassaddiq, A., Abouzaid, M.S.: Certain generalized fractional integral inequalities. AIMS Math. 5(2), 1588-1602 (2020)

33. Nisar, K.S., Tassaddiq, A., Rahman, G., Khan, A.: Some inequalities via fractional conformable integral operators. J. Inequal. Appl. 2019, 217 (2019)

34. Podlubny, I.: Fractional Differential Equations. Mathematics in Science and Engineering, vol. 198. Academic Press, San Diego (1999)

35. Qi, F., Rahman, G., Hussain, S.M., Du, W.S., Nisar, K.S.: Some inequalities of Čebyšev type for conformable $k$-fractional integral operators. Symmetry 10, 614 (2018). https://doi.org/10.3390/sym10110614

36. Rahman, G., Nisar, K.S., Mubeen, S., Choi, J.: Certain inequalities involving the $(k, \rho)$-fractional integral operator. Far East J. Math. Sci.: FJMS 103(11), 1879-1888 (2018)

37. Rahman, G., Nisar, K.S., Qi, F.: Some new inequalities of the Gruss type for conformable fractional integrals. AIMS Math. 3(4), 575-583 (2018)

38. Rahman, G., Ullah, Z., Khan, A., Set, E., Nisar, K.S.: Certain Chebyshev type inequalities involving fractional conformable integral operators. Mathematics 7, 364 (2019)

39. Sarikaya, M.Z., Aktan, N., Yildirim, H.: On weighted Chebyshev-Gruss like inequalities on time scales. J. Math. Inequal. 2(2), 185-195 (2008)

40. Set, E., Akdemir, A.O., Çelik, B.: On generalization of Fejér type inequalities via fractional integral operator. Filomat 32(16), 5537-5547 (2018)

41. Set, E., Çelik, B.: Certain Hermite-Hadamard type inequalities associated with conformable fractional integral operators. Creative Math. Inform. 26(3), 321-330 (2017) 
42. Set, E., Choi, J., Çelik, B.: Certain Hermite-Hadamard type inequalities involving generalized fractional integral operators. Rev. R. Acad. Cienc. Exactas Fís. Nat., Ser. A Mat. 112(4), 1539-1547 (2018)

43. Set, E., Mumcu, I.., Demirbaş, S.: Conformable fractional integral inequalities of Chebyshev type. Rev. R. Acad. Cienc Exactas Fís. Nat., Ser. A Mat. 113, 2253-2259 (2019). https://doi.org/10.1007/s13398-018-0614-9

44. Set, E., Noor, M.A., Awan, M.U., GÖzpinar, A.: Generalized Hermite-Hadamard type inequalities involving fractional integral operators. J. Inequal. Appl. 2017, 169 (2017)

45. Tassaddiq, A.: A new representation of k-gamma functions. Mathematics 7, 133 (2019)

Submit your manuscript to a SpringerOpen ${ }^{\odot}$ journal and benefit from:

- Convenient online submission

Rigorous peer review

- Open access: articles freely available online

- High visibility within the field

- Retaining the copyright to your article

Submit your next manuscript at $\gg$ springeropen.com 